\title{
Coronary Lesion by Angiographic Finding
}

National Cancer Institute

\section{Source}

National Cancer Institute. Coronary Lesion by Angiographic Finding. NCI Thesaurus. Code C119201.

An angiographic finding of pathologic material in a coronary vessel, which obstructs myocardial perfusion. 